\title{
Anabases
}

ANABASES Traditions et réceptions de l'Antiquité

$4 \mid 2006$

Varia

\section{« Daremberg et Saglio » ou « Saglio et Pottier »? La difficile gestation d'un dictionnaire savant}

\section{Catherine Valenti}

\section{(2) OpenEdition}

1 Journals

Édition électronique

URL : http://journals.openedition.org/anabases/2969

DOI : 10.4000/anabases.2969

ISSN : 2256-9421

Éditeur

E.R.A.S.M.E.

\section{Édition imprimée}

Date de publication : 1 octobre 2006

Pagination : 159-167

ISSN : 1774-4296

\section{Référence électronique}

Catherine Valenti, « «Daremberg et Saglio » ou « Saglio et Pottier » ? La difficile gestation d'un

dictionnaire savant », Anabases [En ligne], 4 | 2006, mis en ligne le 01 janvier 2012, consulté le 21

octobre 2019. URL : http://journals.openedition.org/anabases/2969; DOI : 10.4000/anabases.2969

(c) Anabases 


\section{«Daremberg et Saglio" ou «Saglio et Pottier" ? La difficile gestation d'un dictionnaire savant}

Catherine VALENTI

L paradoxe, qui tient autant au parcours scientifique de ses deux principaux concepteurs qu'à la façon dont l'ouvrage est passé à la postérité. Le premier paradoxe est que ni Daremberg ni Saglio ne sont au départ des historiens de l'Antiquité : Daremberg est médecin, tandis que Saglio possède une formation de juriste. D’autre part, ce dictionnaire des Antiquités est souvent désigné comme "Le Daremberg " alors même que le premier fascicule est paru plusieurs années après la mort de Charles Daremberg. Si ce dernier est bien le concepteur et l'initiateur du Dictionnaire, le véritable directeur de la publication est en réalité Edmond Saglio, assisté, à partir de 1884 par l'antiquisant Edmond Pottier. Aussi dès la mort de Saglio en 1911 se trouve-t-il de nombreuses voix, dans le milieu des spécialistes de l'Antiquité, pour affirmer qu'il faudrait plutôt parler $\mathrm{du}$ " Daremberg, Saglio et Pottier ", voire du "Saglio et Pottier », Daremberg n'ayant eu qu'un rôle d'impulsion. Leur plaidoyer n'a apparemment pas été entendu puisque l'on parle encore couramment aujourd'hui du "Daremberg et Saglio ", voire du «Daremberg " tout court.

Ce paradoxe apparent rend compte en réalité de la difficile gestation de l'ouvrage : il s'est écoulé près de trente ans entre le moment où l'idée d'élaborer un Dictionnaire des Antiquités grecques et romaines a germé dans l'esprit de Daremberg, et la date de la première parution effective. Dans la genèse de cette formidable entreprise, le voyage en Italie effectué par Daremberg en 1849-50 revêt une importance particulière : il en rapporte en effet le projet du Dictionnaire. Sa mise en œuvre est plus laborieuse, depuis le contrat signé en 1855 avec les éditions Hachette jusqu'aux débuts de la collaboration avec Edmond Saglio, à partir du milieu des années 1860. Après la mort de Daremberg en 1872, Saglio, assisté du jeune helléniste Edmond Pottier, sollicite de nombreux collaborateurs et supervise la parution des fascicules : effective à partir de 1877 , elle se 
poursuit jusqu'en 1919. C'est cette lente élaboration du «Daremberg et Saglio » ou du "Saglio et Pottier» qu'il s'agit de retracer ici.

\section{Daremberg : des années de formation au voyage en Italie}

\section{La formation : un médecin passionné d'Antiquité}

Charles Daremberg est un enfant naturel, né à Dijon en 1817 . Il est élève du petit séminaire de Saint-Bernard de Plombières-lès-Dijon, puis entreprend des études de médecine, d'abord à Dijon, puis à Paris où il devient docteur en 1841. C'est au cours de ses études que son goût pour l'Antiquité s'est fait jour, notamment un intérêt passionné pour la médecine antique sous l'influence de son maître Emile Littré (1801-1881), qui fut son mentor très tôt dans sa carrière. Spécialiste d'Hippocrate, Littré avait lui-même poursuivi des études de médecine mais refusé de soutenir sa thèse pour des raisons idéologiques. Daremberg pour sa part est un fervent admirateur de Galien auquel il consacre sa thèse de 1841 sous le titre Exposition des connaissances de Galien sur l'anatomie, la physiologie et la pathologie du système nerveux. Daremberg travaille avec passion et obstination, " complétant ses connaissances littéraires et médicales par des études d'anatomie comparée au Muséum d'histoire naturelle auprès de savants aussi renommés que Blainville, Gratiolet, Duvernoy, Serres, Isidore Geoffroy-Saint-Hilaire, Emmanuel Rousseau, Jacquart ou Rouget ${ }^{1}$ ".

Danielle Gourévitch, grande spécialiste de Daremberg, considère qu'à partir de sa thèse sur Galien en 1841, rien ne peut plus détourner le nouveau docteur en médecine de sa vocation historique. Pour gagner sa vie, Daremberg accepte un poste de médecin des écoles et des bureaux de bienfaisance, et devient bibliothécaire de l'Académie de médecine, puis de la Bibliothèque Mazarine ; mais il entreprend en parallèle dès les années 1840 des missions d'exploration consacrées à la recherche de manuscrits anciens, missions en général financées par le ministère de l'Instruction publique et des Cultes. En 1845 et 1848, il effectue ainsi deux voyages, l'un en Angleterre, l'autre en Allemagne ${ }^{2}$. C'est au cours d'un troisième voyage, en Italie cette fois, que va germer en lui l'idée d'un Dictionnaire des Antiquités grecques et romaines.

1 Danielle Gourévitch, "Charles-Victor Daremberg (1817-1872) et une histoire positiviste de la médecine ", École pratique des Hautes études, sans date. Instituto nazionale di Archeologia e storia dell'arte/ Centre Jean-Bérard - Institut français de Naples, 1994, p. 13. 


\section{Le voyage en Italie (1849-1850)}

C'est en effet à l'occasion de ce voyage, en 1849-1850, que Charles Daremberg forme le projet du Dictionnaire des Antiquités. Il se rend en Italie dans un contexte bien particulier, celui de l'intervention française contre la république romaine : chassé du pouvoir par les révolutions de 1848, le pape Pie IX, réfugié à Gaète, a lancé un appel aux quatre principales puissances catholiques, Autriche, Espagne, France et Royaume de Naples, pour la destruction de la République romaine et le rétablissement de son pouvoir temporel. Bien que bon catholique lui-même, Daremberg ne suit que de très loin les problèmes politiques du moment. D'après Danielle Gourévitch, si son ami Littré peut être qualifié de " gauchiste exalté ", Daremberg lui-même se définirait plutôt comme un " chrétien de gauche "; la révolution de février 1848 l'a passablement inquiété, et il n'adhère en réalité ni à l'esprit révolutionnaire ni à la répression ${ }^{3}$. Il observe l'actualité " avec un libéralisme poli ${ }^{4}$ » et décide de profiter de la présence française à Rome pour effectuer une nouvelle mission scientifique, dans le but de collationner des textes médicaux antiques.

Pour cette entreprise il s'associe à Ernest Renan qu'il a rencontré quelques mois auparavant et qui, bien que de six ans son cadet, est son maître en sciences orientales. Les deux hommes sont passablement gênés par l'aspect politique de l'affaire, mais se justifient en rappelant qu'il y avait aussi des savants dans les armées napoléoniennes. Ainsi le 13 août 1849, Renan écrit-il à sa sœur : "Toutes les armées de Napoléon furent ainsi accompagnées de savants, et Geoffroy Saint-Hilaire ne se fit pas scrupule de mettre à profit pour la science la plus injuste des guerres, celle d'Espagne ${ }^{5}$. " Le projet de Daremberg et Renan est officiellement présenté par une commission de l'Académie des Inscriptions et Belles Lettres lors de la séance du 25 août 1849 et, en septembre suivant, le ministère de l'Instruction publique et des Cultes autorise les deux jeunes gens à partir : Daremberg doit recueillir et collationner les textes des médecins de l'Antiquité ; quant à l'orientaliste Renan, il est chargé d'étudier les manuscrits orientaux ${ }^{6}$. Un arrêté ministériel du 2 octobre 1849 précise encore la mission des deux jeunes savants : ils se voient confier " une mission scientifique et littéraire en Italie, ayant pour objet l'exploration dans les bibliothèques de Rome et des principales villes d'Italie, des manuscrits relatifs à l'histoire et à la littérature médicale dans l'Antiquité et

3 Danielle GOURÉVITCH, “ Charles-Victor Daremberg (1817-1872) et une histoire positiviste de la médecine", art. cit.

4 Danielle GourévitCH, La mission de Charles Daremberg en Italie (1849-1850), op. cit., p. 13.

5 Cité dans Gourévitch, La mission de Charles Daremberg en Italie (1849-1850), op. cit., p. 14.

6 “Mission scientifique en Italie de MM. Daremberg et Renan”, Archives des Missions scientifiques, 1849, $1^{\text {er }}$ cahier, p. 54-73. 
au Moyen Âge, ainsi que la recherche des manuscrits grecs et orientaux, intéressans (sic) pour l'histoire générale et pour les études philologiques 7 ".

Or le voyage de Daremberg en Italie est décisif pour l'achèvement de sa formation intellectuelle. Daremberg a d'énormes lacunes culturelles dont il prend justement conscience lors de son séjour, au cours de " promenades romaines " effectuées en compagnie de Renan ${ }^{8}$. Il se rend compte également que ces manques ne sont pas seulement les siens, mais ceux de la science française en général : la France au milieu du XIX siècle ne possède pas de grand dictionnaire des antiquités pouvant rivaliser avec les ouvrages allemands et anglais ${ }^{9}$. En ce sens, le voyage en Italie constitue bien la matrice intellectuelle du Dictionnaire des Antiquités : dès son retour en France, fort de ce constat, Daremberg élabore le projet et prend des contacts en vue de sa réalisation.

\section{Les débuts du « Daremberg et Saglio »}

\section{Le contrat avec Hachette}

Le 23 juillet 1855 est signé un contrat entre Charles Daremberg et Louis Hachette. L'originalité du projet " tient à la fois dans son titre comme dans les clauses du premier contrat 10 ". L'œuvre est présentée comme universelle - le contrat évoquant un "Dictionnaire universel" -, et il faut comprendre cet adjectif dans une double acception, spatiale et technique, au sens premier du terme. Les antiquités orientales " côtoient les antiquités plus classiques (grecques et romaines), mais il s'agit aussi de présenter les civilisations concernées sous tous leurs aspects, comme le spécifie l'article $1^{\text {er }}$ du contrat rédigé ${ }^{11}$ »: «M. Daremberg se charge de rédiger, tant par lui-même qu'avec des collaborateurs de son choix, pour la librairie de MM. Hachette et Cie, un Dictionnaire universel des Antiquités orientales, grecque, latine et du Moyen Âge, comprenant $1^{\circ} \mathrm{La}$ religion, les lois et les institutions politiques, civiles et domestiques, $2^{\circ}$ Les mœurs, usages et coutumes, $3^{\circ}$ Les langues écrites et parlées, $4^{\circ}$ Les arts et métiers (l'architecture, la statuaire, la peinture, la glyptique, les monnaies, la céramique, la métallurgie, les ameublements), l'épigraphie, la paléographie, etc., $5^{\circ}$ Les monuments conservés ${ }^{12}$."

7 Lettre du ministre de l'Instruction publique et des Cultes à Charles Daremberg, 2 octobre 1849, conservée à l'Académie de Médecine, 125/537, nº 932.

Danielle Gourévitch, La mission de Charles Daremberg en Italie (1849-1850), op. cit., p. 36.

$9 \quad$ Ibid.

10 Philippe JockeY, L'archéologie, Paris, Belin, 1999, p. 135.

11 Ibid.

12 Cité dans Danielle Gourévitch, La mission de Charles Daremberg en Italie (1849-1850), op. cit., p. 36. 
Le dictionnaire est là pour "vulgariser ", au sens restreint du terme : il s'agit de " toucher, au-delà des savants, l'honnête homme, amateur d'antiquités, d'arts, de lettres 13 ". L'édition " grand public " tire ainsi à son tour les conséquences de la révolution du regard savant porté sur les civilisations passées, et " place les données matérielles au rang de vecteur de la connaissance ${ }^{14}{ }$. On peut noter cependant qu'en ce qui concerne le Daremberg, l'universalité revendiquée dans le premier contrat ne sera pas totalement respectée, puisqu'en 1863 les antiquités chrétiennes feront finalement l'objet d'une publication à part avec le Dictionnaire des Antiquités chrétiennes de l'abbé Martigny 15 - Daremberg pour sa part ne fera jamais totalement son deuil de la scission des antiquités chrétiennes ${ }^{16}$.

Au point de vue de la forme, le Dictionnaire des Antiquités grecques et romaines adopte finalement le modèle du dictionnaire anglais d'Antony Rich, A Dictionary of Roman and Greek Antiquities, publié pour la première fois en 1858 : adoption du vocabulaire latin, forme des articles avec références aux auteurs, vignettes semées dans le texte. On pourrait s'étonner de voir un ouvrage de 1858 servir de modèle au Daremberg dont la conception remonte pourtant à la fin des années 1840 : c'est que la gestation du projet a été particulièrement lente. L'entreprise est en effet colossale, et ne peut être menée à bien par le seul Daremberg. Comme le prévoyait le contrat passé avec Louis Hachette, Daremberg s'est adjoint un certain nombre de collaborateurs, et notamment Edmond Saglio qui rejoint l'aventure vers le milieu des années 1860 et va y jouer un rôle de premier plan, surtout après la mort de Daremberg en 1872.

\section{L'arrivée de Saglio}

Saglio lui non plus n'est pas au départ un historien de l'Antiquité. Né à Paris en 1828, il entreprend des études de droit ; après avoir décroché sa licence, il est attaché quelque temps à la Commission des grâces du ministère de la Justice puis devient avocat. Mais d'après Henri Omont, auteur de l'éloge funèbre de Saglio prononcé en 1911 devant l'Académie des Inscriptions et Belles Lettres, "là n'était pas sa voie 17 ". Sa véritable vocation, il la découvre bientôt en suivant les cours de Jules Quicherat, et se donne alors « sans réserve à l'histoire de l'art et à l'archéologie 18 ». Or Quicherat est un médiéviste,

13 Philippe JockeY, L'archéologie, op. cit., p. 135.

14 Ibid.

15 Cf. Danielle GourÉVITCH, "Un épisode de l'histoire du Dictionnaire des Antiquités connu sous le nom de 'Daremberg et Saglio' : la publication du Dictionnaire des Antiquités chrétiennes de l'abbé Martigny ”, Caesarodunum, tome XXVII, 1993.

16 Ibid., p. 85.

17 Henri Omont, " A l'occasion de la mort de M. Edmond Saglio ", Comptes Rendus de l'Académie des Inscriptions et Belles Lettres, séance du 8 décembre 1911, p. 2. Ibid. 
spécialiste d'archéologie médiévale ${ }^{19}$, et son influence sur Saglio a été effectivement marquante : en 1861, Edmond Saglio entre au Louvre comme conservateur adjoint du département de la sculpture moderne et des objets d'art du Moyen Âge et de la Renaissance - il sera directeur de ce département de 1879 à 1893 , puis deviendra directeur du Musée de Cluny de 1893 à $1903^{20}$.

C'est pourtant à ce spécialiste de l'art médiéval que Daremberg fait appel en 1865 ; cette année-là, les deux hommes commencent à correspondre, et Saglio est rapidement associé au projet du Dictionnaire des Antiquités grecques et romaines. Outre la direction générale qu'il assume d'abord avec Daremberg, Saglio rédige un grand nombre d'articles spéciaux relatifs aux ustensiles, aux meubles, aux vêtements, à la mythologie, sujets pour lesquels il était particulièrement compétent. Saglio recrute également d'autres rédacteurs, notamment Guillaume et Heuzey pour l'architecture, Humbert et Baudry pour le droit romain. Le premier volume du Dictionnaire des Antiquités grecques et romaines ne paraît cependant qu'en 1877, soit cinq ans après la mort de Daremberg et treize ans après la mort de Louis Hachette, les deux concepteurs du projet - le tout premier volume est daté de 1873 , mais la date est fictive car ce premier tome de 1873 n'a jamais été mis en vente ${ }^{21}$. Ainsi dès la fin du XIXe siècle certains se sont interrogés sur la dénomination du Dictionnaire des Antiquités : fallait-il parler du "Daremberg et Saglio " ou plutôt du "Saglio et Pottier ", en référence à Edmond Pottier qui rejoint le projet en 1884 et y prend une place déterminante ?

\section{III. « Daremberg et Saglio » ou « Saglio et Pottier » ?}

\section{Le "Dictionnaire des Antiquités " de la mort de Daremberg à l'arrivée de Pottier (1872-1884)}

Daremberg a indéniablement donné l'impulsion : c'est lui qui a eu l'idée du dictionnaire, c'est lui qui a signé le contrat avec Louis Hachette, c'est lui également qui en 1857 a dressé le plan général de l'ouvrage conservé dans un registre de la Sorbonne ; dans ce texte Daremberg précise notamment les limites chronologiques de l'ouvrage : « Pour toutes ces antiquités notre Dictionnaire commence avec les temps historiques et s'arrête au moment où le monde ancien cède définitivement la place à un monde

19 Ève Gran-AYMERICH, Dictionnaire biographique d'archéologie 1798-1945, Paris, CNRS éd., 2001, p. 555. 1999, tome II, p. 1271. tiennes de l'abbé Martigny”, art. cit., p. 79. 
nouveau, c'est-à-dire aux environs du VIIe siècle ${ }^{22} \ldots$.. Si le rôle de Daremberg est évidemment primordial, le travail véritable a toutefois été accompli par Saglio assisté d'un grand nombre de spécialistes de l'Antiquité.

Il faut distinguer en réalité deux périodes dans l'élaboration du "Daremberg et Saglio ». Entre 1872 et 1884, Saglio, à cause de la mort de Daremberg, se retrouve de facto le seul directeur de publication. Saglio s'est énormément investi dans le projet, au point que certains, à la mort de Saglio en 1911, estiment que l'on ne devrait pas parler $\mathrm{du}$ " Daremberg et Saglio » mais bel et bien du "Saglio et Pottier ». Toutes les notices nécrologiques consacrées à Saglio insistent sur le rôle central de ce dernier - bien plus que de Daremberg - dans l'élaboration du Dictionnaire des Antiquités. "Ce grand ouvrage ", écrit par exemple le chanoine Chevalier, auteur en 1913 d'une Notice sur la vie et les travaux de M. Edmond Saglio, " a été pendant plus de cinquante ans la pensée centrale de la vie de M. Saglio et l'aboutissement de tous ses travaux 23 "; d'après Henri Omont, Saglio a été "l'homme d'un seul livre ", le Dictionnaire des Antiquités 24 . Le chanoine Chevalier fait l'éloge, hagiographie oblige, de l'inlassable patience de Saglio dans la gestion des collaborateurs du dictionnaire : "Avec une douceur jamais lassée, [Saglio] a écrit des milliers de lettres pour rappeler les promesses oubliées, faire ressortir les inconvénients de[s] retards qui paralysaient l'œuvre entière ${ }^{25}$. "

Ainsi l'élaboration du dictionnaire semblait-elle nécessiter, tout autant que des compétences scientifiques, des talents de diplomate dont savait apparemment faire preuve Saglio : "Il se trouvait parfois dans la pénible nécessité de retirer un article à celui qui l'avait accepté et de chercher un nouvel auteur : il y fallait beaucoup de diplomatie ${ }^{26}$. "Saglio peine néanmoins pendant cette première période à trouver des spécialistes aptes à rédiger les différents articles du dictionnaire, et doit alors se charger lui-même d'une partie notable de la rédaction. Dans les premiers fascicules, il est ainsi l'unique auteur de plusieurs centaines d'entrées ; aussi le cheminement dans l'alphabet se révèle-t-il particulièrement lent.

À partir de 1884, la situation du Dictionnaire des Antiquités s'améliore considérablement : cette année-là en effet, Saglio s'associe à l'helléniste Edmond Pottier, décrit par le chanoine Chevalier comme " un savant de la jeune école 27 ».

Sorbonne, ms 150 .

Notice sur la vie et les travaux de M. Edmond Saglio, par M. le chanoine Ulysse CHEVALIER, membre de l'Académie, lue dans la séance du 16 mai 1913, Paris, Académie des Inscriptions, 1913, p. 37.

Ibid.

Ibid., p. 37. 


\section{L'arrivée de Pottier et l'achèvement du projet}

En 1884, Edmond Pottier est effectivement un jeune savant. Âgé de 29 ans, il a passé trois ans à l'École française d'Athènes entre 1877 et 1880, avant d'être nommé à son retour en France maître de conférences de littérature grecque à la Faculté des lettres de Rennes. En 1883 il occupe le même poste à la Faculté des lettres de Toulouse où il passe son doctorat. En 1884, l'année où il s'associe à Saglio, Pottier vient d'être nommé suppléant à l'École des Beaux Arts à Paris et conservateur au Louvre où il entre comme " attaché libre » au Département des antiquités orientales et de la céramique ${ }^{28}$.

Pottier est donc un ancien "Athénien ", arrivé à Athènes à la fin de la direction d'Albert Dumont. Or Dumont, directeur de l'École française pendant un peu moins de trois ans, de 1875 à 1878, a réformé en profondeur l'institution " athénienne ", la dotant d'un Bulletin de Correspondance hellénique et créant un Institut du même nom. Son action s'inscrit dans un contexte particulier : celui d'une rivalité franco-allemande révélée par le conflit franco-prussien de 1870-1871 et exacerbée dans les années suivantes par une intense propagande nationaliste. Ces tensions nouvelles trouvent un prolongement sur les chantiers de fouilles de la Grèce, et ce d'autant plus que la science allemande représente depuis longtemps pour les savants français un modèle à imiter mais aussi, si possible, un idéal à surpasser.

Ce sont ainsi les séquelles du conflit franco-allemand qui contribuent à orienter l'École d'Athènes vers l'archéologie : «Après 1870, l'admiration pour l'Allemagne intellectuelle se transforme en volonté de l'égaler et même de la dépasser sur ses terrains d'excellence 29 ", notamment l'histoire antique et la philologie. Pottier fait partie du groupe des "mamelouks " d'Albert Dumont ${ }^{30}$, et on peut supposer qu'à partir de 1884, il va mettre en œuvre les leçons apprises à Athènes en faisant du Dictionnaire des Antiquités grecques et romaines, tout autant qu'un outil de travail incomparable, l'élément d'une revanche scientifique sur l'Allemagne.

C'est sous l'égide de ces deux hommes qu'une génération sépare, Edmond Saglio et Edmond Pottier, que les fascicules du Dictionnaire des Antiquités paraissent désormais régulièrement à partir de 1884 . Pottier prend alors une part active à la direction du dictionnaire en même temps qu'il assure à l'ouvrage le concours de nombreux rédacteurs. D'autre part, Saglio lui-même a formé un autre savant : il s'agit de son gendre Georges Lafaye, qui le remplace d'ailleurs comme directeur de publication à sa mort en 1911. Les derniers fascicules du "Daremberg et Saglio » continuent à paraître jusqu’à l'achèvement de l'alphabet en 1919.

Ève GRAN-AYMERICH, Dictionnaire biographique d'archéologie 1798-1945, op. cit., p. 544545. 1998, p. 205. 
On peut estimer avec Philippe Jockey que le "Daremberg et Saglio ", ou le "Saglio et Pottier ", voire le " Daremberg, Saglio et Pottier " pour réconcilier tous les points de vue, par son ambition, son ampleur et son étendue, constitue « l'ancêtre lointain des encyclopédies sur CD-ROM 31 ». Destiné à contrer la concurrence étrangère et notamment allemande, le « Daremberg et Saglio » fait des émules en Europe dès la fin du XIXe siècle : en Italie commence à partir de 1886 la publication du Dizionario epigrafico di antichità romana; quant aux Allemands, ils répliquent au "Daremberg " dès 1894 avec la monumentale Realencyclopädie der Altertumswissenschaft. Les Anglo-saxons dans le même temps font paraitre plusieurs dictionnaires sur l'Antiquité classique. Si le contenu du "Daremberg et Saglio " mérite d'être mis à jour et ses problématiques renouvelées par les apports de la sociologie et de l'anthropologie, le Dictionnaire des Antiquités grecques et romaines reste inégalé dans la maîtrise des sources textuelles. Il constitue d'autre part un jalon marquant de l'historiographie des études classiques en France.

\begin{tabular}{l} 
CATHERINE VALENTI \\
\hline Université de Toulouse II - Le Mirail \\
UFR d'Histoire, Arts et Archéologie \\
5, allées Antonio Machado \\
F-31058 Toulouse \\
cathy.valenti@wanadoo.fr
\end{tabular}

31 Philippe Jockey, L’archéologie, op. cit., p. 135. 